\title{
EMOTION REGULATION AND GENDER
}

\author{
Emna Bouguira, Uta Ouali, Yosra Zgueb, Rabaa Jomli, Fethi Nacef \\ Department of Psychiatry A. Razi Hospital, La Manouba, Tunisia \\ Email address: uta.ouali@gmail.com, bouguiraemna9@gmail.com
}

Objectives : To study the relationship between gender and emotion regulation strategies.

Background and aims : Since early age, population tend to stereotype genders. Research shows that gender differences in the psychological domain have a socio-cultural, but also a biological basis [1]

Neurocognitive studies showed differences on emotional maturity and brain development between male and female during the period of puberty, adolescence and late adolescence [2]. Little is still known about the way gender impacts individual's regulation of emotion.

The aim of our study was to evaluate the relationship between emotion regulation strategies and gender.

Materials and methods : This is a cross-sectional study conducted between February and April 2017 at the Faculty of Legal Science and an Engineering School (ESPRIT) in Tunis/Tunisia. Students filled in the validated Tunisian-Arabic version of the Regulation of Emotions Questionnaire-2 (REQ-2T) as well as a questionnaire containing socio-demographic and clinical variables. Statistical analysis was performed using SPSS- 20.

Results : $\mathbf{3 8 4}$ students took part in the study. Mean age of participants was 22 years. The study showed an equal distribution between genders: 162 (42,3\%) males, 221 (57,7\%) females. The study showed a significant relationship between male gender and external dysfunctional emotion regulation strategies as well as between female gender and external functional emotion regulation strategies.

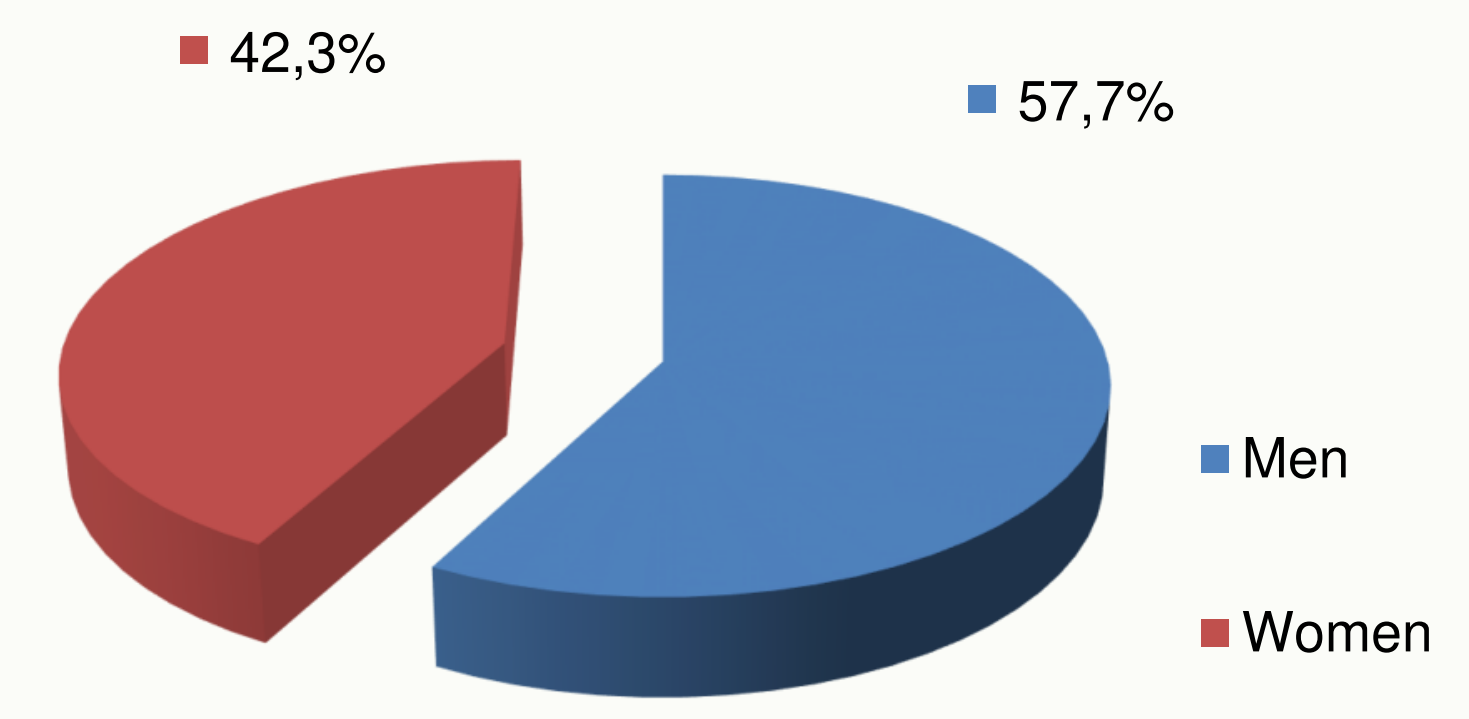

Chart 1: Distribution of participants by gender
Discussion : Men used non-adatative external regulatory strategies. Differences in gender regulation strategies are also reported in literature and demonstrate the importance of introducing, from an early age, education in emotional regulation away from stereotypical, patriarchal models $[1,3]$.

New studies in neurocognitives sciences show that the quality of emotional regulation is partly related to maturity and brain development [2]. Emotional regulation involves judgment of the situation, memorization of previous situations and experiences, and the ability to regulate the expression of emotions. These three abilities essential to emotion regulation are intimately linked to the respective development of the prefrontal cortex, limbic system, and cortical and subcortical maturity [4].

Differences in pubertal initiation and cerebral development between genders may also explain differences in emotion regulation strategies during adolescence and late adolescence $[3,4]$.

Conclusions : Gender seemed to impact emotion regulation strategies. This can be explained both by education and neurocognitive developmental differences between genders. Therefore, early education to emotion regulation strategies and decreasing stereotypes are necessary to address this problem.

The authors declare that there is no conflict of interest regarding the study.

\section{Table I: Statistical correlations between the REQ-2T subscales and the student's gender (using the non-parametric Mann Whitney test)}

\begin{tabular}{ccccc}
\hline & Men & Women & Significance \\
\hline $\begin{array}{c}\text { Internal dysfunctional } \\
\text { emotion regulation } \\
\text { strategies (F1) }\end{array}$ & Median & 12,00 & 12,00 & 0,853 \\
\hline $\begin{array}{c}\text { Internal functional } \\
\text { emotion regulation } \\
\text { strategies (F2) }\end{array}$ & Median & 18,00 & 17,00 & 0,826 \\
$\begin{array}{c}\text { External dysfunctional } \\
\text { emotion regulation } \\
\text { strategies (F3) }\end{array}$ & Median & 7,00 & 6,00 & 0,001 ** \\
\hline $\begin{array}{c}\text { External functional } \\
\text { emotion regulation } \\
\text { strategies (F4) }\end{array}$ & Median & 10,00 & 12,00 & 0,000 ** \\
\hline
\end{tabular}

1. Morris AS, Silk JS, Steinberg L, Myers SS, Robinson LR. The role of the family context in the development of emotion regulation. Soc Dev. 2007;16(2):361-88.

2. . Ahmed SP, Bittencourt-Hewitt A, Sebastian CL. Neurocognitive bases of emotion regulation development in adolescence. Dev Cognit Neurosci. 2015;15(1):15-25. 